\title{
Green fluorescent protein as a novel species- specific marker in enteric dual-species biofilms
}

\author{
Lucy C. Skillman, ${ }^{1}$ lan W. Sutherland, ${ }^{1}$ Martin V. Jones ${ }^{2}$ \\ and Amanda Goulsbra
}

Author for correspondence: Lucy C. Skillman. Tel: +44131650 5325. Fax: +441316505392. e-mail: lucy.skillman@ed.ac.uk

1 Institute of Cell and Molecular Biology, University of Edinburgh, Mayfield Road, Edinburgh EH9 3JH, UK

2 Unilever Research, Port Sunlight Laboratory, Quarry Road East, Bebington, Wirra L63 3JW, UK

\begin{abstract}
Green fluorescent protein (GFP) was used as a tool to examine the interactions between pairs of bacterial species and their effects on subsequent biofilm development over $\mathbf{2 4}$ h. A plasmid encoding GFP from Aequorea victoria was transformed into strains of Enterobacter agglomerans and Escherichia coli ATCC 11229. The development of dual-species biofilms, containing one fluorescent and one non-fluorescent partner, was examined using viable counts. UV illumination of plates enabled both species to be identified in a mixture. The spatial distribution of each species was examined by UV microscopy, simultaneously staining the non-fluorescent strain with propidium iodide. GFP fluorescence was measured to quantify the adhesion of the strains to other cells or cell constituents or the invasion into pre-existing biofilms. Cooperation between Ent. agg/omerans/GFP and Klebsiella pneumoniae G1 resulted in a 54 and a $\mathbf{2 3} \%$ increase in biofilm formation, respectively, compared with single-species biofilms. E. coli/GFP and Serratia marcescens 87b stably co-existed in biofilms but did not affect the growth of each other. The other bacterial partnerships examined were competitive, with the end result that one species dominated the biofilm. The methods described provide a convenient technique for the examination of mixed-species biofilm communities where the unique interactions between species determine the true properties of the resultant biofilms.
\end{abstract}

Keywords: green fluorescent protein (GFP), mixed biofilm, adhesion, co-operation, competition

\section{INTRODUCTION}

Green fluorescent protein (GFP) was originally isolated from the jellyfish Aequorea aequorea (Shimomura et al., 1962) and the cDNA of GFP was cloned from Aequorea victoria by Prasher et al. (1992). The use of GFP has expanded and it has rapidly become a widely used reporter of gene regulation (Cubitt et al., 1995; Crameri et al., 1996). GFP is a 238 aa protein which emits green light $(508 \mathrm{~nm})$ when excited by blue light $(395 \mathrm{~nm})$. It can be detected by irradiation with near-UV or blue light and requires no substrate or cofactors for activity. Chalfie et al. (1994) first described the use of cloned GFP to monitor gene expression in both prokaryotes and eukaryotes. Vectors for use in Gram-negative bacteria

Abbreviations: GFP, green fluorescent protein; PI, propidium iodide. other than Escherichia coli have now been constructed (Matthysse et al., 1996). GFP has been used to investigate a range of diverse systems from host-pathogen interactions to activated sludge communities (Valdivia et al., 1996; Eberl et al., 1997; Leff \& Leff, 1996; Niswender et al., 1995; Albano et al., 1996). More recently, Bloemberg et al. (1997) have reported the use of GFP plasmids to identify Pseudomonas species in a biofilm and on seedling roots.

In most natural and industrial environments, biofilms are complex communities consisting of more than one microbial species. A two-species system is simple enough to allow quantitative analysis and in situ speciation (Camper et al., 1996; Stewart et al., 1997; Banks \& Bryers, 1991; Siebel \& Characklis, 1991). Diversity in microbial communities leads to a variety of complex relationships involving interspecies and intraspecies interactions. The interactions between organisms within 
plaque have been comprehensively studied and found to be both beneficial and antagonistic (Hasty et al., 1992; Marsh et al., 1997; Whittaker et al., 1996). McEldowney \& Fletcher (1987) studied the sequential attachment of a number of bacteria and found that biofilm development depended on species composition, surface composition and the sequence of attachment. Brading et al. (1997) investigated the challenge of a monoculture biofilm of one organism with another. Although Pseudomonas fluorescens had advantages for initial colonization over Pseudomonas putida, neither species precluded the incorporation of the other species into the biofilms. Thus specific surface interactions between different species are thought to play a major role in controlling succession during the development of a microbial community. Specific, direct interactions may involve adhesins on the bacterial cell surface and receptors on the surface of other bacterial cells of the same or different species (James et al., 1995). Investigation of the specific adhesins on the bacterial cell surface, on which the inhibition or reversal of some coaggregations occurs with the addition of simple sugars, suggests lectin-like proteins can be involved (Kinder \& Holt, 1994).

The species investigated in this study are all members of the Enterobacteriaceae; three were isolated from an industrial biofilm and identified as Enterobacter agglomerans, Klebsiella pneumoniae and Serratia marcescens. They show between 60 and $7 \%$ genetic relatedness, with Ent. agglomerans and $K$. pneumoniae G1 the most closely related and $S$. marcescens $87 \mathrm{~b}$ and Ent. agglomerans the most distant. The presence of enteric bacterial species in industrial environments may present considerable problems of hygiene and monitoring. This is accentuated when the bacteria are present in biofilms because of their increased resistance to disinfection. The aim of this work was to compare interactions between these species and to correlate them with increased or decreased biofilm formation, using plasmid-encoded GFP to label and subsequently identify Ent. agglomerans/GFP and E. coli/GFP.

\section{METHODS}

Bacterial strains. Enterobacter agglomerans, Klebsiella pneumoniae G1 and Serratia marcescens $87 \mathrm{~b}$ were isolated from biofilms on industrial surfaces and typed using the API 20E identification system (bioMérieux). E. coli ATCC 11229 is a strain commonly used for industrial testing purposes. The organisms were cultured in yeast extract (YE) medium (Sutherland \& Wilkinson, 1965). The strains had doubling times $(\ln 2 / \mu)$ of: $90 \mathrm{~min}, K$. pneumoniae; $75 \mathrm{~min}$, Ent. agglomerans/GFP; $60 \mathrm{~min}, S$. marcescens; and $55 \mathrm{~min}, E$. coli/GFP. Non-fluorescent and fluorescent strains were coupled to allow subsequent differentiation using viable counts, microscopy and fluorimetry.

Transformation by electroporation. The strains Ent. agglomerans and E. coli ATCC 11229 were grown up in $100 \mathrm{ml} \mathrm{YE}$ to early exponential phase, transferred to a chilled tube and incubated with ice for $15 \mathrm{~min}$. The cells were pelleted at $2700 \mathrm{~g}$ for $10 \mathrm{~min}$ at $4{ }^{\circ} \mathrm{C}$, washed twice in $10 \mathrm{ml} 1 \mathrm{mM}$ HEPES, $\mathrm{pH} 7.0$, and resuspended in $0.5 \mathrm{ml}$ cold $10 \%$ glycerol. Sus- pension $(0.2 \mathrm{ml})$ was mixed with $1 \mu \mathrm{l}$ pBAD DNA (Crameri et al., 1996) in TE buffer $(1 \times$ TE is $10 \mathrm{mM}$ Tris $/ \mathrm{HCl}, 1 \mathrm{mM}$ EDTA). This was electroporated in a cold $0.2 \mathrm{~cm}$ cuvette applying one pulse $(2.5 \mathrm{kV}, 25 \mu \mathrm{F}, 200 \Omega)$. The cells were resuspended in SOC (recovery) buffer (2\% Bacto-tryptone, $0.5 \%$ Bacto yeast extract, $10 \mathrm{mM} \mathrm{NaCl}, 10 \mathrm{mM} \mathrm{MgCl}_{2}$, $10 \mathrm{mM} \mathrm{MgSO}, 2.5 \mathrm{mM} \mathrm{KCl}, 20 \mathrm{mM}$ glucose), incubated at $30{ }^{\circ} \mathrm{C}$ for $1 \mathrm{~h}$ and plated out onto agar containing ampicillin and $0.5 \%$ arabinose. pBAD-GFP transformants could then be selected on the basis of their ampicillin resistance and GFP expression could be induced with $0.5 \%$ arabinose and visualized using UV illumination. The resultant transformants will be referred to as Ent. agglomerans/GFP and E. coli/GFP and their growth rates were the same as the non-transformed strains.

Plasmid stability. YE broth (without arabinose or ampicillin; $100 \mathrm{ml}$ ) was inoculated with $1 \times 10^{5}$ Ent. agglomerans/GFP (or E. coli/GFP) cells and incubated, shaking at $30^{\circ} \mathrm{C}$ for $24 \mathrm{~h}$. One millilitre was then dispensed into fresh broth and incubated for a further $24 \mathrm{~h}$. This was repeated for a total of $72 \mathrm{~h}$. At each time interval, samples were removed, serially diluted and plated out. Loss of the plasmid could be calculated by counting the non-fluorescent colonies. To further monitor plasmid stability, a $1 \mathrm{l}$ chemostat vessel containing $700 \mathrm{ml}$ YE $+1 \%$ glucose was inoculated with Ent. agglomerans/GFP. Different flow rates were used to assess plasmid loss at different doubling times. Samples were taken at steady state after three residence times $\left(T_{\mathrm{R}}=1\right.$ /dilution rate), diluted and plated out.

Properties of GFP. Sphaeroplasts (prepared as outlined by Osborn et al., 1972) were sonicated to assess the effect of cell lysis on fluorescence. The sphaeroplasts were progressively broken by sonication for $4 \times 1 \mathrm{~min}$ bursts. The fluorescence of sonicated samples was measured on a Perkin Elmer LS 50B fluorimeter. A crude extract of GFP was prepared by centrifuging an overnight culture of Ent. agglomerans/GFP at $5000 \mathrm{~g}$ for $10 \mathrm{~min}$. The pellet was resuspended in $10 \mathrm{ml}$ PBS (Oxoid), $50 \mu \mathrm{l}$ of a protease inhibitor (PMSF) was added and it was sonicated for $4 \mathrm{~min}$. It was recentrifuged at 15000 r.p.m. for $20 \mathrm{~min}$ to remove membranes and other cell debris, resulting in a crude protein preparation. The protein was then used to investigate the effect of $\mathrm{pH}$ and temperature on protein denaturation and fluorescence. Twenty microlitres of protein $\left(1 \mathrm{mg} \mathrm{ml}^{-1}\right)$ or whole cells was mixed with $80 \mu \mathrm{PBS}$ at the appropriate $\mathrm{pH}$ and fluorescence was determined.

Biofilm growth and invasion studies. Biofilms were grown on borosilicate glass beads; $25 \mathrm{~g}$ 4-mm-diameter beads was placed in a $250 \mathrm{ml}$ flask with $100 \mathrm{ml} \mathrm{YE}$ and $1 \%$ glucose. Flasks were inoculated in triplicate to obtain approximately $10^{5}$ bacteria $\mathrm{ml}^{-1}$, consisting of one species or a 1:1 mixture of the two species. The flasks were incubated at $30^{\circ} \mathrm{C}$ with shaking at 80 r.p.m. Six beads were removed from the three flasks, and each bead was rinsed and transferred to an Eppendorf tube containing $1 \mathrm{ml} \mathrm{PBS}$, vortexed for $30 \mathrm{~s}$ to remove the biofilm cells, diluted and plated out. Fluorescent colonies (Ent. agglomerans/GFP and E. coli/GFP) were identified in a mixture by UV illumination of the plates. For microscopy, glass coverslips were used for biofilm growth in a batch culture system consisting of a box containing YE broth $(250 \mathrm{ml})$ and $1 \%$ glucose. The coverslips were supported by a metal holder. A small magnetic stirring bar was also incorporated in the system and a magnetic stirrer set at 100 r.p.m. provided thorough mixing of available nutrients. Each system was inoculated with overnight bacterial cultures to obtain approximately $10^{5}$ bacteria $\mathrm{ml}^{-1}$. The invasion of 
non-fluorescent biofilm monolayers by a second fluorescent partner was investigated using fluorimetry. Aliquots $(100 \mu \mathrm{l})$ of $K$. pneumoniae or $S$. marcescens overnight cultures were added to each well of a microtitre plate and incubated at $30^{\circ} \mathrm{C}$ for $16 \mathrm{~h}$. The liquid phase was removed and $100 \mu \mathrm{l}$ overnight cultures of E. coli/GFP or Ent. agglomerans/GFP were added and incubated at $30^{\circ} \mathrm{C}$. At $1,2,4$ and $24 \mathrm{~h}$, the excess liquid was removed, the wells were washed once with PBS and $100 \mu \mathrm{l}$ PBS was added. The fluorescence of the biofilms in the wells was measured on a Perkin Elmer LS 50B fluorimeter. Adhesion to plastic surfaces was lower than adhesion to glass for all strains. This did not affect enhanced dual-species biofilm formation by Ent. agglomerans/GFP and K. pneumoniae.

Microscopy. Sixteen hour dual-species biofilms on glass coverslips were immersed in a detergent $(10 \mathrm{mM}$ cetylpyridinium chloride) for $5 \mathrm{~min}$, then immersed in propidium iodide (PI; $25 \mu \mathrm{g} \mathrm{ml}^{-1}$, Sigma). Pretreatment with the detergent caused cell membrane damage and access of PI to the cell interior. GFP was not masked by the presence of PI in the cell, therefore Ent. agglomerans/GFP cells appeared green (or yellow) and $K$. pneumoniae cells appeared red under UV illumination. The biofilms were observed on a Polyvar microscope with a tungsten bulb attachment (Reichert-Jung) with a violet-blue excitation filter $(395-446 \mathrm{~nm})$. This method gave no indication of cell viability but was effective for examining spatial distribution.

Production of inhibitors. Stationary phase liquid cultures of $E$. coli/GFP and $S$. marcescens were centrifuged at $10000 \mathrm{~g}$ for $10 \mathrm{~min}$ and the supernatant was filter-sterilized. Aliquots $(50 \mu \mathrm{l})$ of the $S$. marcescens or E. coli/GFP supernatants were added to $50 \mu \mathrm{l}$ YE in each well of a microtitre plate. $K$. pneumoniae or Ent. agglomerans/GFP stationary phase liquid culture supernatant $(50 \mu \mathrm{l})$ was used for controls. Stationary phase $K$. pneumoniae $\left(\mathrm{OD}_{570} 0.25 ; 25 \mu \mathrm{l}\right)$ was added to $E$. coli/GFP supernatant and $25 \mu \mathrm{l}$ Ent. agglomerans/GFP was added to $S$. marcescens supernatant. The increase in turbidity was measured after $24 \mathrm{~h}$ incubation at $30^{\circ} \mathrm{C}$ on a Beckman MR 5000 plate reader. Inhibition of growth indicated the presence of a growth inhibitor in the supernatant.

Adhesion. Ent. agglomerans/GFP and K. pneumoniae cooperated, forming biofilms more successfully when together than apart, indicating specific interactions. The interactions were investigated by measuring adhesion and its inhibition, using fluorescence (Ent. agglomerans/GFP) and turbidity ( $K$. pneumoniae). Ent. agglomerans/GFP cells were treated with a protease [bromelain; 103 units ( $\mu$ g protein) ${ }^{-1} \mathrm{~h}^{-1}$ ] and a polysaccharide-degrading enzyme obtained from an Enterobacter-specific bacteriophage to inhibit the adhesion. Phagefree enzyme was prepared by filtration using a $100 \mathrm{kDa}$ filter (Vivaspin; Vivascience). The preparation had a specific activity of $3.78 \times 10^{-3} \mu \mathrm{mol}$ glucose $\mathrm{h}^{-1}(\mathrm{mg} \text { protein })^{-1}$. EDTA was used to determine the effect on adhesion of ion sequestration. Ent. agglomerans/GFP overnight culture $(10 \mathrm{ml})$ was added to $10 \mathrm{ml}$ sterile distilled $\mathrm{H}_{2} \mathrm{O}$ (control), $10 \mathrm{ml} 2 \mathrm{mg}$ EDTA ml ${ }^{-1}$ or $200 \mu \mathrm{g}$ EDTA ml ${ }^{-1}$. One hundred microlitres of each was added to each $K$. pneumoniae monolayer and allowed to attach for $5,15,30,45,60,90$ and $120 \mathrm{~min}$. The liquid phase was removed and the wells were rinsed once with PBS. The fluorescence of adhering Ent. agglomerans/GFP was then measured on a Perkin Elmer LS 50B fluorimeter.

In addition to the ability to measure Ent. agglomerans/GFP adhesion using fluorescence measurements, a method has been developed to enable the adhesion of $K$. pneumoniae (a nonfluorescent strain) to be quantified. The technique relies on the ability of $K$. pneumoniae to re-grow into the nutrient-rich liquid phase above the biofilm. To prevent re-growth of the base species (Ent. agglomerans/GFP), it was killed using UV irradiation for $120 \mathrm{~min}$ prior to K. pneumoniae addition. Aliquots $(100 \mu \mathrm{l})$ of washed stationary phase Ent. agglomerans/GFP cells were added to each well of a transparent microtitre plate and incubated at $30^{\circ} \mathrm{C}$ for $24 \mathrm{~h}$. The Ent. agglomerans/GFP monolayers were killed by exposing to $\mathrm{UV}$ for $120 \mathrm{~min}$. K. pneumoniae cells $(100 \mu \mathrm{l})$ were added for the required time. The wells were then rinsed once with PBS, which was replaced with $100 \mu \mathrm{l}$ YE (+ glucose). The regrowth of $K$. pneumoniae into the liquid phase was proportional to the number of attached cells. The adhesion of $K$. pneumoniae could thus be measured using a Dynatech MR 5000 plate reader to quantify the increase in turbidity. To inhibit $K$. pneumoniae adhesion to Ent. agglomerans/GFP, $10 \mathrm{ml}$ stationary phase $K$. pneumoniae cells was treated with bromelain and various commercial polysaccharase mixtures. In addition, $0.5 \%$ mannose was added during a $30 \mathrm{~min}$ attachment period of $K$. pneumoniae to Ent. agglomerans/ GFP monolayers. Re-growth after $20 \mathrm{~h}$ was measured on a Dynatech 5000 plate reader.

\section{RESULTS AND DISCUSSION}

\section{Use of GFP as a species-specific marker}

Usually the plasmid encoding GFP can be selected for by including a gene encoding antibiotic resistance. In this system, plasmid maintenance in the absence of ampicillin selection is an important factor because when mixed cultures are used, the antibiotic may adversely affect the growth of the second species. After $72 \mathrm{~h}$ in batch culture, $0.5 \%$ of Ent. agglomerans/GFP cells had lost their plasmid. The plasmid loss in E. coli/GFP was higher : after $72 \mathrm{~h} 14 \%$ of cells had lost the plasmid. In continuous culture, plasmid loss increased with increasing specific growth rate (Table 1). The length of biofilm experiments was therefore kept to a minimum, i.e. below $24 \mathrm{~h}$ with a maximum plasmid loss of $4 \cdot 6 \%$ (E. coli/GFP) and $0.2 \%$ (Ent. agglomerans/GFP). Sonication of Ent. agglomerans/GFP sphaeroplasts leading

\section{Table 1. Loss of the GFP plasmid from Ent. agglomerans/GFP in chemostat culture in the absence of ampicillin selection}

Samples were plated out onto YE agar containing arabinose $(0.5 \%)$ and UV illumination of the plates enabled nonfluorescing colonies to be identified. SE values are presented where $n=4$.

\begin{tabular}{|ccc|}
\hline $\begin{array}{l}\text { Doubling } \\
\text { time (h) }\end{array}$ & $\begin{array}{c}\text { Plasmid } \\
\text { loss (\%) }\end{array}$ & SE \\
\hline 0.5 & $28 \cdot 6$ & $1 \cdot 90$ \\
2.5 & $21 \cdot 8$ & $1 \cdot 50$ \\
4.5 & 16.2 & $2 \cdot 10$ \\
6.5 & $12 \cdot 2$ & $1 \cdot 80$ \\
8.5 & 4.3 & 0.50 \\
10.5 & 0.5 & 0.05 \\
\hline
\end{tabular}



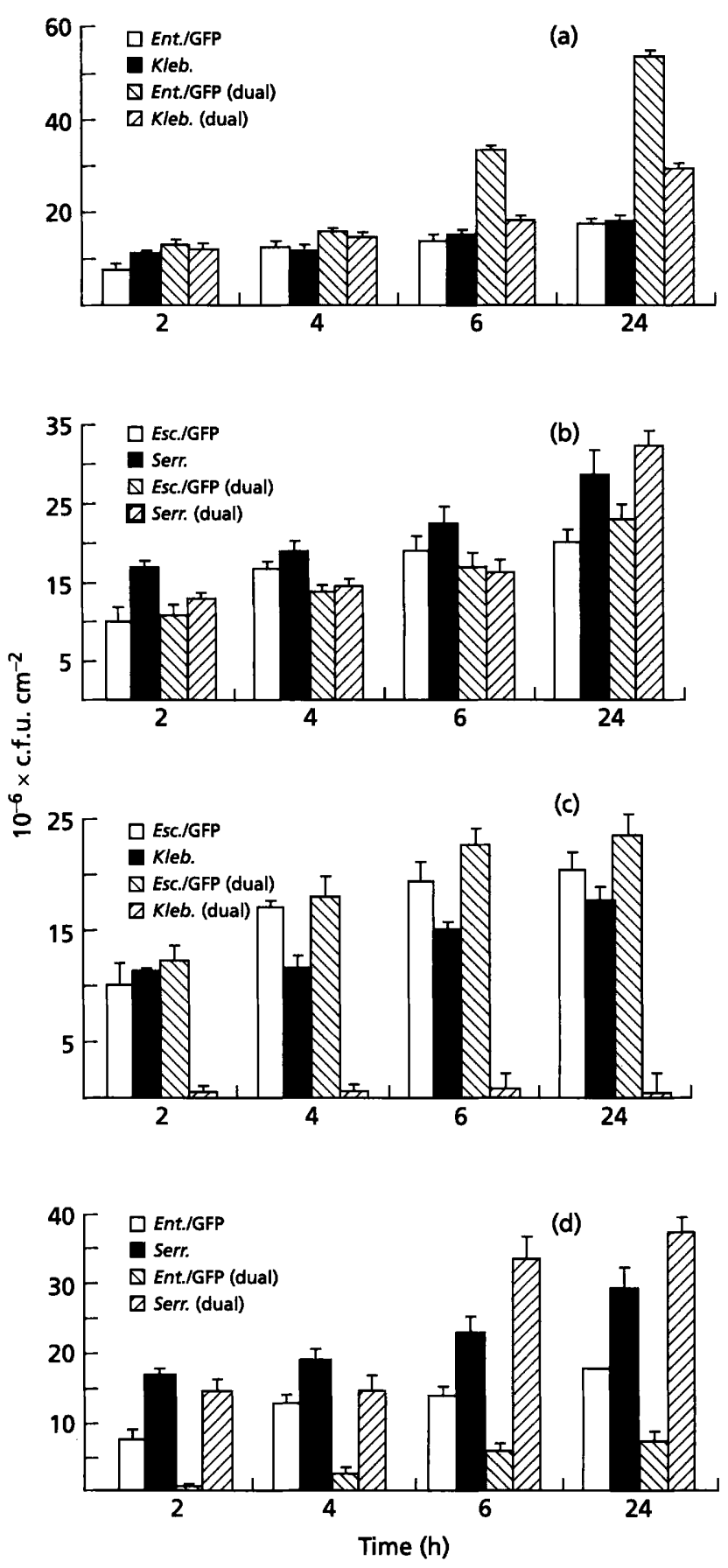

Fig. 1. Comparison of single-species and dual-species biofilm development on glass beads using viable counts. Two strains, $E$. coli/GFP and Ent. agglomerans/GFP, were transformed with a plasmid encoding GFP. Colonies in a mixture could then be identified using UV illumination of plates. Results are presented as surface coverage $\left(\mathrm{cm}^{-2}\right)$. (a) Ent. agglomerans/GFP $+K$. pneumoniae; (b) E. coli/GFP $+S$. marcescens; (c) E. coli/GFP $+K$. pneumoniae; (d) Ent. agglomerans/GFP +S. marcescens. Bars represent SE, where $n=6$.

to progressive cell lysis did not result in a large decrease in fluorescence. A larger decrease in fluorescence was observed between 10 and $30 \mathrm{~min}$ after sonication, possibly due to cellular enzyme degradation. This has important implications because fluorescence can only be related to the presence of GFP, not intact cells. In addition, dead cells will still fluoresce if they are killed after synthesizing GFP.

A crude extract of the protein was isolated from Ent. agglomerans/GFP and compared with intact cells to determine the stability of the protein. GFP was more sensitive to low $\mathrm{pH}$ than to high $\mathrm{pH}$, with maximum fluorescence occurring at $\mathrm{pH} 8-10$. The effect of $\mathrm{pH}$ was muted in whole cells. In addition, the protein was thermostable up to $60^{\circ} \mathrm{C}$. Because the GFP is so stable and persists after cell death, fluorescence per se cannot be used to indicate viability. However, fluorescence can be used to assess adhesion of Ent. agglomerans/GFP cells and microscopic localization is possible. GFP is a cytoplasmic protein with low toxicity and should therefore have a minimal influence on bacterial cell surface dynamics. The interactions between bacterial species should not be adversely affected.

\section{Dual-species biofilm formation}

Single- and dual-species biofilm formation between the four enterobacterial species was monitored by viable counts over $24 \mathrm{~h}$ (Fig. 1). A total inoculum of $1 \times 10^{5}$ cells ml $\mathrm{m}^{-1}$ of one species or a 1:1 mixture of the two organisms was used. Viable cell numbers in singlespecies biofilms were greatest for $S$. marcescens, followed by E. coli/GFP, K. pneumoniae and Ent. agglomerans/GFP. The biofilm growth of the four dualspecies biofilms, comprising one fluorescent and one non-fluorescent partner, differed according to the species involved. In dual-species biofilms of K. pneumoniae and Ent. agglomerans/GFP, both showed increased adherence and growth when compared with single-species biofilms : Ent.agglomerans/GFP showed a $54 \%$ increase and $K$. pneumoniae a $23 \%$ increase in $24 \mathrm{~h}$ dual-species biofilms. The co-operation could be the result of specific adhesion mechanisms increasing their attachment. This correlates with another study on dual-species biofilms containing Salmonella enteritidis and K. pneumoniae, which also showed synergistic biofilm formation, with increased attachment and metabolic activity (Jones \& Bradshaw, 1997). E. coli/GFP and $S$. marcescens did not affect the attachment or growth of each other: cell numbers were the same whether or not the other partner was present. They did not compete and this could indicate that they have separate binding sites or mechanisms of attachment. Stewart et al. (1997) have also reported a system where $K$. pneumoniae and Pseudomonas aeruginosa stably coexisted in biofilms despite differing growth rates under planktonic conditions. Both Ent. agglomerans/GFP $+S$. marcescens and $E$. coli/GFP $+K$. pneumoniae showed competitive interactions. $S$. marcescens out-competed Ent. agglomerans/GFP, with Ent. agglomerans/GFP comprising a maximum $14.7 \%$ of the biofilm. E. coli/GFP out-competed $K$. pneumoniae, with $K$. pneumoniae comprising between 0.6 and $3.1 \%$ of the biofilm. 


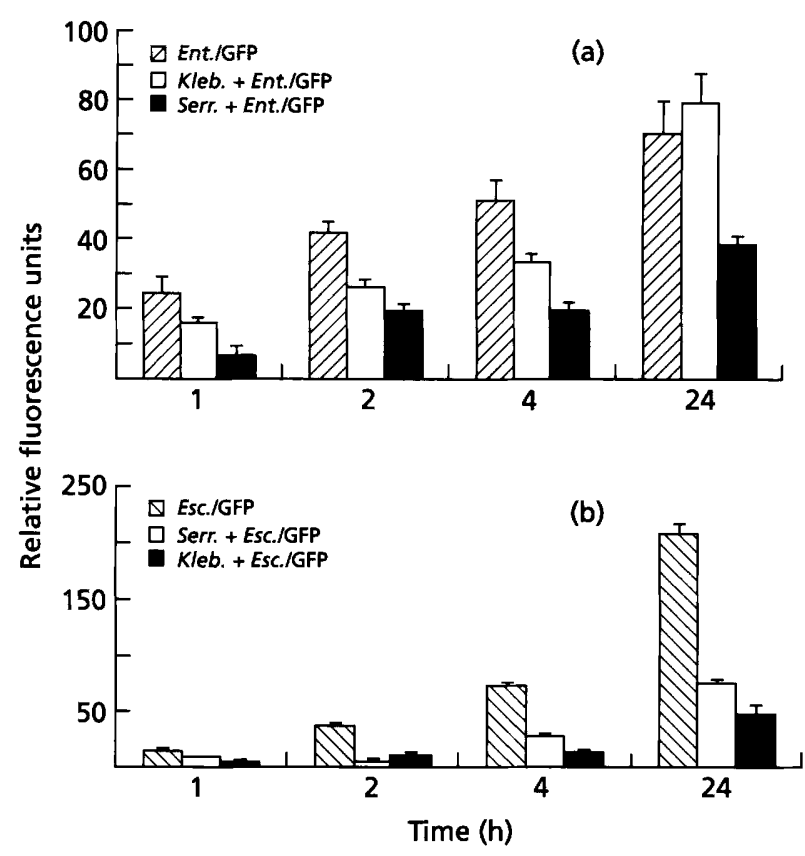

Fig. 2. Invasion of bacterial monolayers of non-fluorescent strains ( $K$. pneumoniae and $S$. marcescens) by fluorescent strains labelled with a plasmid encoding GFP. The incorporation of E. coli/GFP and Ent. agglomerans/GFP into the monolayers was measured, after various contact times, on a Perkin Elmer LS 50B fluorimeter. (a) Ent. agg/omerans/GFP invasion into $K$. pneumoniae and S. marcescens monolayers; Ent. agglomerans/GFP adhesion to blank wells was included as control. (b) $E$. coli/GFP invasion into $K$. pneumoniae and $S$. marcescens monolayers; $E$. coli/GFP adhesion to blank wells was included as control. Bars represent SE, where $n=84$.

\section{Invasion and interactions}

Incorporation of the fluorescent strains into pre-existing biofilms was quantified after various time intervals (Fig. 2). E. coli/GFP was better at binding to blank wells than Ent. agglomerans/GFP. Ent. agglomerans/GFP successfully invaded the K. pneumoniae biofilm, with numbers almost as high (or higher at $24 \mathrm{~h}$ ) as for uncolonized surfaces. Ent. agglomerans/GFP was incorporated at lower levels into $S$. marcescens monolayers, representing on average $19 \%$ of the cells bound to blank wells. $E$. coli/GFP did not invade biofilms of $S$. marcescens or $K$. pneumoniae very successfully, representing on average 35 and $21 \%$ of cells bound to blank wells, respectively. This demonstrated that invasion was not dependent on biofilm-forming ability; for example, when inoculated simultaneously, E. coli/GFP out-competed $K$. pneumoniae and $S$. marcescens out-competed Ent. agglomerans/GFP. The resultant biofilms were dominated by the best competitor of the pair. When a monolayer of $K$. pneumoniae was already present, the competitive partner (E. coli/GFP) showed poor integration into the biofilm. In contrast, where cooperative associations between Ent. agglomerans/GFP and $K$. pneumoniae occurred, bacteria could more easily invade a monolayer of their partner. This suggests that

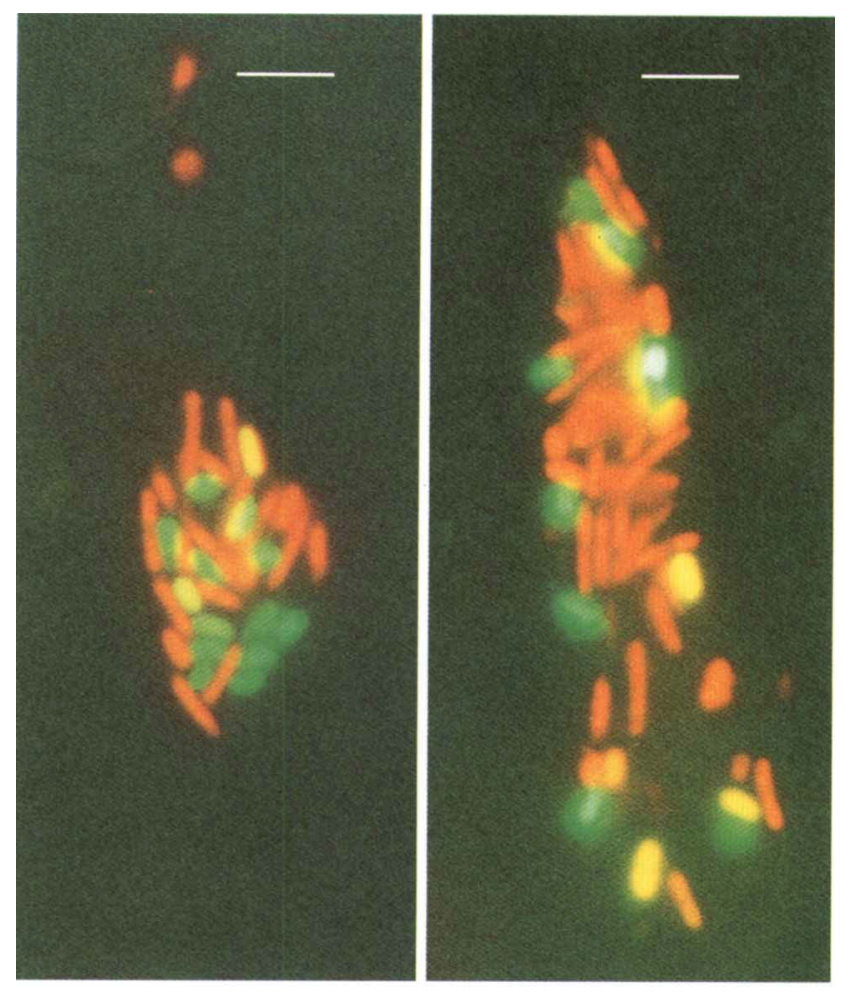

Fig. 3. Microcolonies of a $16 \mathrm{~h}$ co-operative dual-species biofilm of Ent. agglomerans/GFP and $K$. pneumoniae. Biofilms were grown on glass coverslips, and treated with $10 \mathrm{mM}$ cetylpyridinium chloride $(5 \mathrm{~min})$ and $25 \mu \mathrm{g} \mathrm{PI} \mathrm{ml}{ }^{-1}(5 \mathrm{~min})$ before visualizing on a Polyvar microscope with a tungsten bulb attachment and violet-blue excitation filter (395-446 nm). Ent. agglomerans/GFP cells appeared green (or yellow) and $K$. pneumoniae cells appeared red under UV illumination. Bars, $3 \mu \mathrm{m}$.

co-operation is beneficial in terms of invasion. If a bacterial species possesses the ability to adhere specifically to another species it could gain a foothold in a new environment, enhancing its survival. If the cooperation is beneficial, it could in turn help its partner.

GFP is extremely stable and persists up to $60^{\circ} \mathrm{C}$ and within the $\mathrm{pH}$ range 2-12 and also through treatment with formaldehyde, allowing the detection of GFP even in fixed samples (Eberl et al., 1997). This allowed the visualization of Ent. agglomerans/GFP (or E. coli/GFP) cells in dual-species biofilms, even following the detergent treatment necessary to label the second species, $K$. pneumoniae (or S. marcescens), with PI. However, this also prevented the use of GFP as a marker of viability. Microscopic examination revealed that Ent. agglomerans/GFP and K. pneumoniae are often closely associated in microcolonies (Fig. 3). The close proximity suggests that surface-associated macromolecules form the basis of the interactions. This may involve adhesins and receptors on the surface of one or both species. In contrast, E. coli/GFP and S. marcescens mainly formed discrete microcolonies. As $S$. marcescens $+E$. coli/GFP formed biofilms in equal numbers, irrespective of 
Table 2. Inhibition of the adhesion of Ent. agglomerans/GFP cells to monolayers of $K$. pneumoniae, and $K$. pneumoniae cells to Ent. agglomerans/GFP monolayers in microtitre plates

Percentage reduction of Ent. agglomerans/GFP adhesion was calculated after measuring the fluorescence of attached cells. Percentage reduction of $K$. pneumoniae adhesion was calculated after turbidometric measurement of the re-growth of adhering cells. SE values are presented where $n=84$.

\begin{tabular}{|lcc|}
\hline Inhibitor or treatment & $\begin{array}{c}\text { Reduction } \\
\text { in adhesion } \\
(\%)\end{array}$ & SE \\
& & \\
\hline Ent. agglomerans/GFP & & \\
adhesion to K. pneumoniae & $0 \cdot 0$ & - \\
Mannose & $46 \cdot 3$ & $8 \cdot 0$ \\
Protease (bromelain) & $38 \cdot 6$ & $4 \cdot 1$ \\
Specific phage depolymerase & $17 \cdot 5$ & $3 \cdot 3$ \\
EDTA & & \\
K. pneumoniae adhesion to & & \\
Ent. agglomerans/GFP & $5 \cdot 0$ & $0 \cdot 6$ \\
Mannose & $0 \cdot 0$ & - \\
Protease (bromelain) & & \\
Polysaccharase mixtures: & $22 \cdot 3$ & $1 \cdot 1$ \\
Cellulase TV & $10 \cdot 1$ & $1 \cdot 2$ \\
Driselase & $0 \cdot 0$ & - \\
$\beta$-Glucuronidase & $0 \cdot 0$ & - \\
Mutanolysin & $2 \cdot 1$ & $3 \cdot 2$ \\
Hemicellulase & $2 \cdot 6$ & $2 \cdot 1$ \\
Cellulase & & \\
\hline
\end{tabular}

whether the other was present, they interacted 'neutrally' and therefore were not investigated further.

If one species can initially out-compete another species, then it could exploit that particular microenvironment. The production of inhibitors by $S$. marcescens and $E$. coli/GFP, the competitive partners, has also been investigated.

To determine whether the competitive partners ( $E$. coli/GFP and $S$. marcescens) produced bacteriocins, the supernatants were tested for growth inhibition of $K$. pneumoniae and Ent. agglomerans/GFP, respectively. There was no evidence that either species produced any growth inhibitors as growth remained unchanged and cross-streaking on agar plates had no effect. Their dominance in dual-species biofilms was probably due to their higher growth rates. As K. pneumoniae and Ent. agglomerans/GFP formed biofilms co-operatively, their specific interactions were investigated in more detail (Table 2). Protease treatment caused a $46 \%$ reduction in Ent. agglomerans/GFP adhesion to $K$. pneumoniae. Degrading the extracellular polysaccharide with a specific phage depolymerase also caused a 39\% reduction in adhesion. The fluorescence measurements used to follow the adhesion of Ent. agglomerans/GFP to $K$. pneumoniae imply that the interactions are both protein- and polysaccharide-dependent. The extracellular polysaccharides may act as receptors to the adhesins, not only coating the bacterial outer surface but also adsorbing to inert surfaces. This may influence intercellular contacts and accumulation at surfaces. Alternatively, production of extracellular polysaccharide or other cell products by one species may coat the surface and indirectly affect the adhesion of another species. Quantification of $K$. pneumoniae adhesion has also permitted the evaluation of treatments to prevent the adhesion of $K$. pneumoniae to Ent. agglomerans/GFP. Addition of mannose caused a small reduction $(4.5 \%, \mathrm{SE} 0.2)$ in adhesion, which may indicate specific adhesin-carbohydrate interactions, although other components must also be important in the interactions between these two species. Bromelain (protease) treatment caused no significant reduction in adhesion. This contrasts with Ent. agglomerans/GFP adhesion, where protease treatment caused a $50 \%$ reduction in adhesion. This may suggest that only Ent. agglomerans/GFP possesses protein adhesins; alternatively, the protein components in K. pneumoniae may be protected from degradation. The polysaccharase mixtures marketed as cellulase TV (Sigma) and driselase (Sigma) also resulted in a decrease in $K$. pneumoniae adhesion. This suggests that more than one cell component is responsible for the interactions between $K$. pneumoniae and Ent. agglomerans/GFP, but both appear to contribute to the beneficial partnership.

\section{Concluding remarks}

Plasmid-borne GFP was used to investigate dual-species biofilm development. E. coli/GFP and $S$. marcescens stably co-existed in biofilms but did not benefit or antagonize each other. The other pairs, E. coli/GFP $+K$. pneumoniae and Ent. agglomerans/GFP $+S$. marcescens, competed for the surface. The ability of $E$. coli/GFP and $S$. marcescens to out-compete $K$. pneumoniae and Ent. agglomerans/GFP, respectively, may be a result of their higher growth rates. $K$. pneumoniae and Ent. agglomerans/GFP co-operated, resulting in enhanced biofilm formation. Both species were involved in the interactions, which may involve adhesin-receptor interactions as they were partially protein and partially carbohydrate mediated. The methods described provide a convenient technique for the examination of mixed-species biofilm communities where the unique interactions between species within biofilms determine the true properties of the resultant biofilms.

\section{ACKNOWLEDGEMENTS}

L.C.S. acknowledges a BBSRC and Unilever CASE award.

\section{REFERENCES}

Albano, C. R., Randers-Eichorn, L., Chang, Q., Bentley, W. E. \& Rao, G. (1996). Quantitative measurement of green fluorescent protein expression. Biotechnol Tech 10, 953-958. 
Banks, M. K. \& Bryers, J. D. (1991). Bacterial species dominance within a binary culture biofilm. Appl Environ Microbiol 57, 1974-1979.

Bloemberg, G. V., O'Toole, G. A., Lugtenberg, B. J. J. \& Kolter, R. (1997). Green fluorescent protein as a marker for Pseudomonas species. Appl Environ Microbiol 63, 4543-4551.

Brading, M. G., Boyle, J. \& Lappin-Scott, H. M. (1997). Colonisation and interactions of Pseudomonas species in binary culture. In Biofilms: Community Interactions and Control, pp. 73-79. Edited by J. W. T. Wimpenny, P. Handley, P. Gilbert, H. M. Lappin-Scott \& M. V. Jones. Cardiff: Bioline.

Camper, A. K., Jones, W. L. \& Hayes, J. T. (1996). Effect of growth conditions and substratum composition on the persistence of coliforms in mixed-population biofilms. Appl Environ Microbiol 62, 4014-4018.

Chalfie, M., Tu, Y., Euskirchen, G., Ward, W. W. \& Prasher, D. C. (1994). Green fluorescent protein as a marker for gene expression. Science 263, 802-805.

Crameri, A., Whitehorn, E. A., Tate, E. \& Stemmer, W. P. C. (1996). Improved green fluorescent protein by molecular evolution using DNA shuffling. Nat Biotechnol 14, 315-319.

Cubitt, A. B., Heim, R., Adams, S. R., Boyd, A. E., Gross, L. A. \& Tsien, R. Y. (1995). Understanding, improving and using green fluorescent proteins. Trends Biochem Sci 20, 448-455.

Eberl, L., Schulze, R., Ammendola, A., Geisenberger, O., Erhart, R., Sternberg, C., Molin, S. \& Amann, R. (1997). Use of green fluorescent protein as a marker for ecological studies of activated sludge communities. FEMS Microbiol Lett 149, 77-83.

Hasty, D. L., Ofek, I., Courtney, H. S. \& Doyle, R. J. (1992). Multiple adhesins of Streptococci. Infect Immun 60, 2147-2152.

James, G. A., Beaudette, L. \& Costerton, J. W. (1995). Interspecies bacterial interactions in biofilms. J Ind Microbiol 15, 257-262.

Jones, K. \& Bradshaw, S. B. (1997). Synergism in biofilm formation between Salmonella typhimurium and a nitrogen-fixing strain of Klebsiella pneumoniae. J Appl Microbiol 82, 663-668.

Kinder, S. A. \& Holt, S. C. (1994). Coaggregation between bacterial species. Methods Enzymol 236, 254-270.

Leff, L. G. \& Leff, A. A. (1996). Use of green fluorescent protein to monitor survival of genetically engineered bacteria in aquatic environments. Appl Environ Microbiol 62, 3486-3488.

McEldowney, S. \& Fletcher, M. (1987). Adhesion of bacteria from mixed cell suspension to solid surfaces. Arch Microbiol 148, $57-62$.
Marsh, P. D., Bradshaw, D. J., Watson, G. K. \& Allison, C. (1997). Community development in a mixed species oral biofilm. In Biofilms: Community Interactions and Control, pp. 65-70. Edited by J. W. T. Wimpenny, P. Handley, P. Gilbert, H. M. LappinScott \& M. V. Jones. Cardiff: Bioline.

Matthysse, A. G., Stretton, S., Dandie, C., McClure, N. C. \& Goodman, A. E. (1996). Construction of GFP vectors for use in Gram-negative bacteria other than Escherichia coli. FEMS Microbiol Lett 145, 87-94.

Niswender, K. D., Blackman, S. M., Rohde, L., Magnuson, M. A. \& Piston, D. W. (1995). Quantitative imaging of green fluorescent protein in cultured cells: comparison of microscopic techniques, use in fusion proteins and detection limits. J Microsc 180, 109-116.

Osborn, M. J., Gander, J. E., Parisi, E. \& Carson, J. (1972). Mechanism of assembly of the outer membrane of Salmonella typhimurium. Isolation and characterisation of cytoplasmic and outer membrane. J Biol Chem 247, 3962-3972.

Prasher, D. C., Eckenrode, V. K., Ward, W. W., Prendergast, F. G. \& Cormier, M. J. (1992). Primary structure of the Aequorea victoria green fluorescent protein. Gene 111, 229-233.

Shimomura, O., Johnson, F. H. \& Saiga, Y. (1962). Extraction, purification, and properties of aequorin, a bioluminescent protein from the luminous hydromedusan, Aequorea. J Cell Comp Physiol 59, 223-239.

Siebel, M. A. \& Characklis, W. G. (1991). Observations of binary population biofilms. Biotechnol Bioeng 37, 778-789.

Stewart, P. S., Camper, A. K., Handran, S. D., Huang, C.-T. \& Warnecke, M. (1997). Spatial distribution and coexistence of Klebsiella pneumoniae and Pseudomonas aeruginosa in biofilms. FEMS Microbiol Ecol 33, 2-10.

Sutherland, I. W. \& Wilkinson, J. F. (1965). Depolymerases for bacterial extracellular polysaccharides obtained from phageinfected bacteria. J Gen Microbiol 39, 373-383.

Valdivia, R. H., Hromockyj, A. E., Monack, D., Ramakrishnan, L. \& Falkow, S. (1996). Applications for green fluorescent protein (GFP) in the study of host pathogen interactions. Gene 173, 47-52.

Whittaker, C. J., Klier, C. M. \& Kolenbrander, P. E. (1996). Mechanisms of adhesion by oral bacteria. Annu Rev Microbiol 50, 513-552.

Received 30 January 1998; revised 29 April 1998; accepted 12 May 1998. 